\title{
Rabaska
}

Revue d'ethnologie de l'Amérique française

D'Entremont, Laurent. Stories to Remember, 4 v., Pubnico, N.-É., Two Acres Publications, 2005-2012 : vol. 1 : A Collection of timeless stories by a long-time storyteller, ISBN

978-0-969-3432-2-1 ; vol. 2 : More timeless Stories by a long-time storyteller, ISBN 978-0-969-3432-3-3 ; vol. 3 : More timeless Stories by a long-time storyteller, ISBN 978-0-969-3432-4-0 ; vol. 4 : Footsteps in the night. More timeless Stories by a long-time storyteller, ISBN 978-0-969-3432-5-7

\section{Carmen d'Entremont}

Volume 12, 2014

URI : https://id.erudit.org/iderudit/1026807ar

DOI : https://doi.org/10.7202/1026807ar

Aller au sommaire du numéro

Éditeur(s)

Société québécoise d'ethnologie

ISSN

1703-7433 (imprimé)

1916-7350 (numérique)

Découvrir la revue

Citer ce compte rendu

d'Entremont, C. (2014). Compte rendu de [D'EnTREMont, LAUREnT. Stories to Remember, 4 v., Pubnico, N.-É., Two Acres Publications, 2005-2012 : vol. 1 : A Collection of timeless stories by a long-time storyteller, ISBN 978-0-969-3432-2-1 ; vol. 2 : More timeless Stories by a long-time storyteller, ISBN 978-0-969-3432-3-3 vol. 3 : More timeless Stories by a long-time storyteller, ISBN 978-0-969-3432-4-0; vol. 4 : Footsteps in the night. More timeless Stories by a long-time storyteller, ISBN 978-0-969-3432-5-7]. Rabaska, 12, 254-256.

https://doi.org/10.7202/1026807ar d'utilisation que vous pouvez consulter en ligne. 
soigneusement ses illustrations, décelant « un esprit curieux et créatif » (p. 303) chez cet artiste qu'elle connaît bien et qui s'est détaché volontairement de l'idéologie édifiante et patriotique que véhiculent souvent les auteurs « au profit de la recherche formelle et de l'expérimentation plastique » (ibid.). Elle note encore chez lui « la simplification des formes, l'équilibre des masses, le dynamisme des jeux d'opposition entre aplats d'encre noire et espaces réservés » (ibid.). Elle remarque aussi qu'il « s'oriente vers l'étude des effets graphiques obtenus grâce aux lignes énergiques, aux formes stylisées et à la taille blanche, procédé qui le distingue de ses contemporains » (p. 303-304). Même s'il est peu connu, il a contribué, selon Danaux, « à l'autonomisation de la gravure au Québec par rapport à la française » (p. 304) et il lui apparaît « comme l'un des principaux acteurs du développement de la gravure moderne au Québec » (ibid.).

À n'en pas douter, voilà une étude pour le moins remarquable, tant par la qualité de son écriture que par la richesse de son propos. Il n'est pas étonnant que Stéphanie Danaux ait mérité le pris Jacques-Cotnam pour son ouvrage, qui est à la fois un apport important tant à l'histoire de l'iconographie au Québec, qu'à l'histoire littéraire et à l'histoire du livre, entre 1840 et 1940. Cet ouvrage se lit comme un roman tant il est intéressant et pertinent aussi pour l'histoire des idées et des mentalités. J'y ai passé des heures agréables en espérant que l'auteur continue à nous charmer.

AuréLIen BoIvin Université Laval, Québec

D’Entremont, Laurent. Stories to Remember, 4 v., Pubnico, N.-É., Two Acres Publications, 2005-2012 : vol. 1 : A Collection of timeless stories by a long-time storyteller, ISBN 978-0-969-3432-2-1; vol. 2 : More timeless Stories by a long-time storyteller, ISBN 978-0-969-3432-3-3 ; vol. 3 : More timeless Stories by a long-time storyteller, ISBN 978-0-969-3432$4-0$; vol. 4 : Footsteps in the night. More timeless Stories by a long-time storyteller, ISBN 978-0-969-3432-5-7.

Laurent d'Entremont, conteur contemporain, est né à Pubnico-Ouest, Nouvelle-Écosse, en 1941. Les souvenirs de la ferme familiale de son grandpère maternel, Adolphe d'Entremont, où il a été élevé, lui tiennent à cœur. Ses aventures et ses voyages l'ont amené à apprécier son village natal. Depuis plus d'une quarantaine d'années, M. d'Entremont se passionne pour l'histoire locale, domaine auquel il s'intéresse en amateur. Inspiré par la mémoire des gens et de ses expériences de vie, il sauvegarde de l'oubli les souvenirs 
du passé. Au début des années 1970, il publia un premier recueil, The Two Acre [s] Farms, dans le but de consigner les histoires de son grand-père qui était un conteur reconnu. Cet essai l'amena à la publication de quelques autres recueils. Laurent d'Entremont nous offre ici une anthologie en quatre volumes des multiples textes qu'il a rédigés, depuis 1990 pour la plupart, à l'intention du journal The Yarmouth Vanguard, et du Regional Magazine, une publication qui dessert la population de la vallée d'Annapolis. Ses récits s'appuient à la fois sur les témoignages oraux que les villageois lui ont transmis et sur son expérience de vie ; ils reflètent en majorité la première moitié du Xxe siècle. L'auteur note, dans la préface de ses ouvrages, que son but est simple : préserver les histoires locales. En plus de décrire la vie d'un temps qui n'est plus, l'auteur décrit, par cet important témoignage oral, les personnages, les lieux et les événements qui ont marqué sa vie et l'histoire de son peuple jusqu'à nos jours.

Laurent d'Entremont fait connaître quelques personnages importants, dont Adelbert d'Eon, qui a passé sa vie à construire des autobus et à conduire des passagers, Stillman d'Entremont, l'aimable barbier qui était connu par tous, Émile LeBlanc, le légendaire médecin de famille, qui offrit ses services aux villageois pendant plus de quarante ans, Désiré d'Eon, reconnu pour ses nombreuses réalisations, notamment la fondation du journal, Le Courrier de la Nouvelle-Écosse, en 1937, Helen Creighton, une folkloriste anglophone qui est venue au village enregistrer les chansons et les airs de quelques villageois, Robbie d'Entremont, le forgeron ou encore, les multiples sages-femmes de la région. Il présente également les diverses facettes de l'industrie de la pêche, ainsi que les entreprises qui ont joué un rôle important, tels que la première salle de quille, le premier comptoir de crème glacée, les magasins généraux, la boulangerie d'Eon (1886-) et le restaurant Red Cap (1946-). De plus, l'auteur confie plein de chers souvenirs : tantôt il se rappelle des plaisirs de la ferme familiale : la senteur du foin récemment fauché, le son des cloches à bœuf et les déjeuners préparés exclusivement de produits de la ferme ; tantôt des visites des colporteurs, de personnes célèbres, comme le grand photographe Bob Brooks, venu photographier les villageois pour en faire une carte postale, et des touristes américains en été. Plusieurs récits touchent aux intérêts et à la vie personnelle de Laurent d'Entremont ; ils portent, entre autres, sur ses diverses expériences de vie, tels que les emplois qu'il a occupés, les voyages mémorables qu'il a faits au cours des années, les personnes qui l'ont influencé, comme son idole, WilfCarter, grand musicien avec qui il correspondit pendant des années, et le duo comique canadien, Wayne et Shuster, qui ont influencé sa carrière de monologuiste comique. L'auteur prend également l'occasion d'explorer quelques célébrations religieuses et sociales, et de présenter des 
coutumes locales, comme la pratique de manger beaucoup d'œufs à Pâques et celle d'aller dans les bois avec la famille pour couper un arbre pour la fête de Noël. Il documente encore les événements quotidiens importants des villageois, comme l'arrivée du Family Herald, un magazine domestique canadien qui divertissait et informait les familles pendant des années, la venue, en 1925, d'un hydravion Curtiss lors du pique-nique paroissial et les visites régulières de la «shopmobile», un autobus-école qui parcourait les régions rurales pour enseigner les arts industriels aux jeunes adolescents, ainsi que les événements historiques qui les ont marqués, telles que la Deuxième Guerre mondiale, la crise économique de 1929 et l'explosion qui s'est produite le 6 décembre 1917 à Halifax. Enfin, les textes de Laurent d'Entremont touchent à de multiples sujets, entre autres, la langue du milieu, l'histoire des Acadiens, la superstition, la station de radio française locale CIFA, la contrebande et les divertissements.

Les quatre ouvrages sont abordables et de lecture agréable. Sur chaque couverture est reproduite une photo de l'auteur. Les textes, à part ceux qui se trouvent dans le dernier volume, sont présentés en ordre chronologique selon la date de rédaction. Chaque recueil réunit plus de cinquante récits qui, dans l'ensemble, offrent aux lecteurs un aperçu de la vie et du riche patrimoine des habitants d'un village rural acadien. Malheureusement, les œuvres de Laurent d'Entremont n'ont pas pénétré le monde de l'ethnologie au Canada français, probablement parce qu'elles présentent en anglais, la langue de choix de plusieurs Acadiens, des histoires qui étaient souvent racontées en français. Les textes, écrits dans un style personnel, sont vivants ; ils font revivre le passé, surtout des souvenirs d'enfance. Ce qu'on peut toutefois reprocher à l'auteur, c'est qu'il rapporte des faits historiques sans fournir de références complètes et qu'il fournit très peu d'informations sur les témoignages, tous renseignements que les spécialistes jugeraient pertinents. Adressé au grand public, ces recueils exposent des témoignages précieux qui intéresseront ceux qui veulent pénétrer dans la vie de cette petite communauté acadienne.

Carmen d'Entremont

Université Sainte-Anne

Deschênes, Gaston et Pierrette Maurais. Contes et légendes de la Côtedu-Sud. Québec, Septentrion, 2013, 327 p. ISBN 978-2-89448-734-1.

Depuis une dizaine d'années, de nombreux ouvrages parus au Québec ont repris l'abondante littérature du fantastique datant de la période entre 1830 et 1920 environ. Certains titres ne représentent que des réimpressions de textes faisant partie du domaine public, les éditeurs cherchant surtout à 